\title{
Impacted maxillary incisors: Causes, Diagnosis and Management
}

\author{
Jehan Zaib Tanki ${ }^{1}$, Talib Amin Naqash ${ }^{2}$, Atul Gupta $^{3}$, Randhir Singh $^{4}$, Ankush Jamwal $^{5}$ \\ ${ }^{I}$ (Consultant Orthodontics \& Dentofacial Orthopaedics, JLNM Hospital Srinagar J\&K, India) \\ ${ }^{2}$ (Resident Department of Prosthodontics, Government Dental College Srinagar J\&K, India) \\ ${ }_{3}^{3}$ House Surgeon, Government Dental College \&Hospital Srinagar J\&K, India)
}

\begin{abstract}
When the incisors fail to erupt at the normal time, it becomes duty of a clinician to determine the cause and formulate an appropriate treatment plan. Impaction of maxillary permanent incisor is not a frequently case in dental practice, but its treatment is challenging because of its importance to facial esthetics. Although the impaction of a permanent tooth is rarely diagnosed during the mixed dentition period, an impacted central incisor is usually diagnosed accurately when there is delay in the eruption of the tooth. Early diagnosis is very important and interceptive orthodontic treatment could not only improve skeletal mal relationship and eliminate functional interferences, but also may correct disturbances during the eruption. This paper describes the causes, early diagnosis and the orthodontic treatment required in the management of impacted maxillary canines.
\end{abstract}

Keywords: Eruption disturbances, Odontoma, Orthodontic traction.

\section{Introduction}

The maxillary incisors and canines, often referred to as the 'social six', are the most prominent teeth in an individual's smile. They are also the teeth that are on maximum display during speech in most individuals. The normal eruption, position and morphology of these teeth are crucial to facial esthetics and phonetics.

There are very few studies reporting any functional problems associated with missing anterior teeth although some speech difficulties have been reported, particularly with the ' $s$ ' sound ${ }^{1}$. As missing upper incisors are regarded as unattractive this may have an effect on self-esteem and general social interaction and it is important to detect and manage the problem as early as possible ${ }^{2}$.

\section{DEFINITION}

\section{Discussion}

Delayed eruption of maxillary incisors requires monitoring or intervention when

1. There is eruption of contra lateral teeth that occurred greater than six months previously;

2. Both central incisors remain unerupted and the lower incisors have erupted greater than one year previously;

3. There is deviation from the normal sequence of eruption (e.g. lateral incisors erupting prior to the central incisor).

\section{INCIDENCE}

The incidence of unerupted maxillary central incisor in the 5-12 year-old age group has been reported as $0.13 \%$ 3 .

In a referred population to regional hospitals the prevalence has been estimated as $2.6 \%$.

\section{CAUSES}

Literature reveals several causes of failure or delayed eruption of maxillary incisors.

Eruption failure may occur if pathological obstructions, such as supernumerary teeth, odontomas, cysts, develop in the eruptive path of the incisor ${ }^{4}$.

Supernumerary teeth and odontomas are the most common cause: $56-60 \%$ of supernumerary teeth cause impaction of permanent incisors due to a direct obstruction for the eruption ${ }^{5}$.

Eruption failure can also be caused by tooth malformation or dilacerations. Dilacerations occur after trauma to a primary tooth, where the developing permanent tooth bud is damaged due to close proximity to the primary tooth. The degree of damage of the permanent tooth depends on the developmental stage of the tooth in question, as well as the type and direction of the trauma inflicted.

Other possible causes of lack of eruption of maxillary incisors are: ectopic position of the tooth bud, non-vital or ankylosed primary teeth, early extraction (or loss) of deciduous teeth, mucosal barriers in the path of eruption that acts as a physical barrier to eruption, endocrine abnormalities, bone disease ${ }^{6}$. 


\section{DIAGNOSIS}

\section{Clinical examination}

An intra-oral examination should be undertaken to identify retained deciduous teeth, buccal-palatal swelling and availability of suitable space for the incisor ( $9 \mathrm{~mm}$ for a central and $7 \mathrm{~mm}$ for lateral incisors).

Important clinical signs are over-retention of the corresponding primary teeth while the contralateral permanent tooth has already erupted; substantial reduction in the available space for permanent tooth eruption or space closure; rotation and inclination of the adjacent teeth; elevation of the soft tissue of the palatal or labial mucosa depending on the tooth location; absence of a bulge in the buccal sulcus at 1-1.5 years before the expected time of tooth eruption ${ }^{7}$.

The pathognomic sign which indicate impaction of a central incisor is the presence on the arch of the homolateral lateral incisor, as these points to an anomaly in the central incisor eruption process ${ }^{8}$.

Deviation from normal sequence of eruption, e.g. lateral incisors erupt prior to the central incisor, or eruption of adjacent teeth occurred 6 months previously (with both incisors unerupted-lower incisors erupted one year previously), are other signs of delayed eruption of maxillary incisors.

The position of the adjacent teeth in the arch should be noted as well as whether they are upright or tilted toward the missing tooth. This may be helpful in determining the location of the unerupted tooth: once it is close to its normal eruption path the neighboring teeth may tilt, but when the unerupted tooth is far from its normal eruption path the adjacent teeth may close the space in a more bodily kind of movement.

\section{Palpation of the alveolar region}

Palpation is a valuable adjunct in final location of the unerupted tooth; it may also help in the radiographic evaluation? .

On palpation of the area, the clinician is likely to encounter a palatal or labial bulge that will help determine the position of the unerupted tooth. In order to locate the crown of the impacted tooth, we locate a painless, incompressible, palatal or vestibular fibro mucosal protuberance. An edentulous ridge with particularly small mesiodistal and vestibulopalatal dimensions at its summit signifies that the tooth is impacted deeper.

Radiographic assessment

Diagnosis of impacted tooth is verified and its location determined through radiographic evaluation ${ }^{10}$. Panoramic radiograph is considered the standard radiographic first-step examination for treatment planning of impacted teeth because it imparts a low dose while giving the best radiographic survey. This film is useful because it is unique in that it will show the entire dentition as a whole, it may reveal the existence of an impacted tooth, the degree of root resorption for the corresponding primary tooth, and the depth of impaction.

Lateral cephalometric is another film that is particularly useful if there is a supernumerary tooth or dilacerations present because it allows visualization in several dimensions ${ }^{11}$.

To be in a position to recommend the best line of treatment and to plan an appropriate strategy, the orthodontists requires the following information: the exact positions of the crown and root apex of the impacted tooth and the 3-dimensional orientation of its long axis; the proximity of the impacted tooth to the roots of the adjacent teeth; the presence of pathology such as supernumerary teeth, odontomes, apical granulomas or cysts, and their spatial relationship with the impacted tooth; the presence of adverse conditions affecting the adjacent teeth, including root resorption; the 3 dimensional anatomy of the crown and root of the impacted tooth.

CT has proved to be superior to other radiographic method in visualizing bone tissue: 3D CT images clearly show the intraosseus location, inclination and morphology of impacted teeth as well as the distance from adjacent structures.

Due to the highly detailed three-dimension information obtained, computerized tomography is the method of choice for accurately defining the position of an unerupted tooth and identifying any root resorption of adjacent teeth not detectable by other methods. The highly detailed information and the excellent tissue contrast without blurring and overlapping of adjacent structures outweigh the high radiation dose, limited availability, and high cost.

Three-dimensional imagery enables analysis of the precise location and orientation of impacted teeth, their situation relative to obstacles to eruption, their external and internal anatomy, the labial and palatal bone thickness; any resorption of the adjacent teeth or pathological bone loss; the presence or absence of a continuous radiolucent line between the root and the bone (possible ankylosis).

Recently cone-beam CT (CBCT) has been introduced ad a technique dedicated to the imaging of dental and maxillofacial structures. It has one-sixth of the radiation of computed tomography, is more time efficient, more cost effective, and is still able to provide three dimensional images, excellent bone differentiation and an unlimited number of views. Its disadvantages include spatial resolution of subtle structures that is slightly inferior to that of CT and limited representation of soft tissues (due to the lower radiation dose) ${ }^{12}$. 


\section{MANAGEMENT}

Although several different approaches to treatment of unerupted maxillary incisors have been proposed in the literature, a common feature among them is that early diagnosis is critical to the success of the treatment. It is thought that the less time the normal eruption is delayed, the better the outcome. If there has been a loss of space, it is necessary to create space prior to treatment and maintain that space throughout the treatment phase. The treatment possibilities vary from conservative to more aggressive approaches.

The most conservative management would be the extraction of any obstruction, the creation of space, and the observation for spontaneous eruption. $70 \%$ of teeth have been reported to erupt spontaneously after removal of obstruction, without any further treatment ${ }^{13}$. In the case of a supernumerary tooth, it is often possible to remove the supernumerary tooth avoiding damage to the underlying incisor, and allow the incisor to erupt on its own.

In certain cases, orthodontic traction may be required ${ }^{14}$. The surrounding erupted teeth may be used for anchorage to orthodontically move the tooth into the arch following surgical exposure of the unerupted tooth. Patient management can be divided into three phases: pre-surgical orthodontics, exposure and bonding, and post-surgical orthodontics. In the pre-surgical phase, surrounding teeth that will be used as anchorage must be banded or bonded, and sufficient space must be created for the erupted tooth. It is also necessary to remove any obstruction (i.e. supernumerary tooth). The next step is surgical exposure of the unerupted tooth, which can be done using one of three methods. A gingivectomy would be the procedure of choice in the presence of attached gingival and if sufficient tooth structure can be exposed to bond an attachment. At least one half to two thirds of the crown must be uncovered. In patients where there is inadequate attached gingival or if a gingivectomy would compromise the width of attached gingiva, an apically positioned flap is recommended. A third method is the closed eruption technique in which a flap that incorporates the attached gingiva is raised and is fully replaced in its original position after placement of traction devices. If the tooth is impacted in the middle of the alveolus or high in the vestibule near the nasal spine, the closed eruption technique may the treatment of choice. Some teeth are impacted so high in the vestibule that an apically positioned flap is difficult or impossible to employ. In all cases, following exposure, isolation of the tooth is required to facilitate bonding. Isolation and hemostasis can be aided by the use of hemostatic agents like Surgicel or Gel-Foam around the tooth. The crown is then etched with a phosphoric acid gel that will reduce the chances of the etch running into the adjacent tissues. The area is then rinsed and dried, and a bonding agent is placed. Finally, a bracket is light-cured onto the tooth and a ligature wire or a gold chain is attached to the bracket. In the case of the closed technique, the flap is replaced and sutured into its original position. Controversy exists regarding the amount of time the clinician should wait to apply post surgical orthodontic forces after exposure and bonding. Some authors recommend application of force immediately after exposure. Others recommend waiting for one to two weeks. This decision appears to be the personal preference of the clinician. It has been reported that treatment of unerupted maxillary incisors with the closed-eruption technique can produce overall good long-term esthetic results.

As long as there is sufficient space available, a tooth will erupt on its own. However, contemporary orthodontics has several techniques available to expedite this process. An alternative to mechanical traction is the use of magnetic traction. Magnets used in orthodontics are neodymium-iron-boron and produce static magnetic fields. Magnets that contain neodymium only were found to be susceptible to corrosion in the presence of oral fluids, which results in the release of potentially harmful products. The magnets are coated with

parylene (poly-para-xylene) in thin sections to prevent corrosion. Research shows that the static magnetic fields produced by orally placed orthodontic rare earth magnets do not result in any change in pulp or gingival tissue adjacent to the magnets. The technique for the use of magnets for tooth eruption involve using Fuji Ortho LC to bond a $3 \times 3 \times 1 \mathrm{~mm}$ neodymium-iron-boron magnet to the unerupted tooth and a $5 \times 5 \times 2 \mathrm{~mm}$ magnet to a removable appliance. During the eruption process, the magnet in the appliance can be changed to precisely direct the movement of the tooth. Movement should occur approximately at the rate of one millimeter per month. Advantages of using rare earth magnets to aid in tooth eruption include their ability to produce a measured force continuously over long periods of time and the position of the magnet can be manipulated by the clinician so that the tooth can erupt into the ideal position. In addition, few adjustments are necessary throughout the entire process as opposed to the frequent adjustments of mechanical devices. Disadvantages include the fact that the force between two magnets diminishes dramatically as the distance increases. Heat and hot liquids cause the magnets to lose flux, and therefore lose force. Finally, the corrosive properties of neodymium-iron-boron are a disadvantage.

Orthodontic traction may have no effect on bringing the tooth into occlusion if the unerupted tooth has been ankylosed. The possibility exists for an erupting incisor to become ankylosed at some point during the eruption process. There is limited literature regarding treatment in such a situation; however, literature was found regarding treatment of an ankylosed incisor resulting from trauma. Ankylosis occurs when replacement resorption occurs at the innermost layer of the periodontal ligament and possibly at the cementum. The healing process takes place from the adjacent alveolar bone causing ankylosis. In situations where a portion of the 
clinical crown is exposed, early clinical diagnosis includes percussion tests where there is a change in the percussion sound and a marked decrease in the normal physiologic facial-lingual excursion. The clinician must be cautious in using percussion sounds as a method of diagnosis. One must not ignore the possibility of a false negative. In situations where the incisor is still unerupted and is completely submerged, percussion would be impossible. When an incisor has been intruded with resulting ankylosis, the clinician can attempt surgical luxation to break the ankylosis while maintaining periapical blood supply at the same time. This results in an inflammatory reaction that encourages the formation of a new fibrous ligament. Immediate application of orthodontic forces is then placed to prevent the reoccurrence of ankylosis. This technique has limited benefits because re-ankylosis in a luxated tooth, even with immediate orthodontic forces, is frequently seen. Chaushu, Becker, suggest certain guidelines for successful ortho-surgical treatment of an ankylosed tooth. First, proper surgical luxation should be performed which results in class 3 tooth mobility. Second, a heavy extrusive force should be promptly applied. Third, the extrusive force should be maintained at an appropriately high level by frequently renewing the elastic force. Distraction osteogenesis, a technique for forming new bone through bone transport, is also well described in the literature. After surgery, a distraction device is placed. These devices can range from a conventional acrylic splint and an expansion screw that is bonded to the ankylosed incisor, to a single tooth distractor that is surgically placed. A latency period is required prior to initiation of the distraction phase. It is mentioned that vertical distraction could possibly be functionally and esthetically unsatisfactory because of the unidirectional impact of intraoral distraction devices. It is possible that distraction devices need to be used in conjunction with other approaches.

If an impacted incisor is associated with a cyst, a conservative approach is preferred in order to prevent the loss of the incisor. When the preservation of the tooth is desired in a young patient where the lesion is isolated, marsupialization is the treatment of choice. Marsupialization is a procedure that involves the creation of a surgical window in the wall of the cyst. The cystic lining is made continuous with the oral cavity. The opening must be maintained artificially to prevent spontaneous closure. The cyst remains open for several months allowing the cavity to be filled with new bone. The involved teeth are then allowed to spontaneously erupt or are orthodontically brought into the correct position. By utilizing marsupialization, treatment is atraumatic and can prevent psychological and mental trauma ${ }^{15}$.

A final treatment option, which should only be used in cases when the unerupted incisor is severely ectopic or if there is extensive pathology and/or risk to adjacent teeth, is extraction of unerupted incisor. In the case of a dilacerated tooth with an extremely acute angle of dilacerations, it is possible for the labially oriented portion of the root to become impacted on the buccal cortical plate. For this reason, dilacerated incisors are often extracted. It is essential that care be exercised to avoid damage to adjacent teeth during removal. The surgeon also should take precautions to avoid loss of alveolar bone that could adversely affect future treatment possibilities including replacement with a bridge, implant, or orthodontic space closure ${ }^{16}$.

\section{Conclusion}

It is not uncommon for children to present with variations in normal eruptive patterns of the maxillary incisors. By virtue of the location of the maxillary incisors, parents are often discouraged when eruption patterns do not follow the norms. This will usually prompt the parent to seek treatment from an orthodontist in order to prevent psychological ramifications that accompany abnormalities of the anterior maxilla. In order to properly treat these individuals, the clinician must have knowledge of the classification, etiology, and treatment modalities available for eruption disturbances of the maxillary incisors. It is important that a thorough examination is performed in order to obtain an accurate diagnosis. The clinical presentations of these eruption disturbances may prove to be a manifestation of an underlying local or systemic condition. In many cases, there may be no treatment necessary except for the creation of space and allowing the tooth to erupt alone in a matter of time. Other cases require surgical and/or orthodontic interception. Each case must be treated independently in order to formulate the proper treatment plan and to achieve the best possible outcome for each patient.

\footnotetext{
References

[1]. Snow K. Articulatory Proficiency in Relation to Certain Dental Abnormalities. Journal of Speech and Hearing Disorders 1961; 26: 209-12.

[2]. Shaw WC, O’Brien KD, Richmond S, Brook P. Quality control in orthodontics: risk/benefit considerations. Br Dent J 1991; 170: 33-37

[3]. Mac Phee CG. The incidence of erupted supernumerary teeth in consecutive series of 4000 school children. Br Dent J 1935; 58: 5960

[4]. Huber KL, Suri L, Taneja P. Eruption disturbances of the maxillary incisors: a literature review 1. J Clin Pediatr Dent. 2008;32(3):221-230.

[5]. Smailiene D, Sidlauskas A, Bucinskiene J. Impaction of the central maxillary incisor associated with supernumerary teeth: initial position and spontaneous eruption timing. Stomatologija. 2006;8(4):103-107.

[6]. Jones JW. A Medico-legal Review of Some Current UK Guidelines in Orthodontics: A personal View. J Orthod. 1999;26:307-324.

[7]. Becker A. The orthodontic treatment of impacted teeth. 1998.
} 
[8]. Chokron A, Reveret S, Salmon B, Vermelin L. Strategies for treating an impacted maxillary central incisor. Int Orthod. 2010 Jun;8(2):152-176.

[9]. Brin I, Zilberman Y, Azaz B. The unerupted maxillary central incisor: review of its etiology and treatment. ASDC J Dent Child. 1982 Sep-Oct;49(5):352-356.

[10]. Douglas DE. Management of impacted anterior teeth utilizing basic orthodontic principles. ASDC J Dent Child. 1989;56(5):353357.

[11]. Smailiene D, Sidlauskas A, Bucinskiene J. Impaction of the central maxillary incisor associated with supernumerary teeth: initial position and spontaneous eruption timing. Stomatologija. 2006;8(4):103-107

[12]. Chaushu S, Chaushu G, Becker A. The role of digital volume tomography in the imaging of impacted teeth. World $\mathbf{J}$ Orthod. 2004;5(2):120-132.

[13]. Kramer RM. Williams AC. The incidence of impacted teeth. A survey at Harlem hospital. Oral Surg., Oral Med., Oral Pathol, 29: 237-241, 1970 .

[14]. Oliver RG. Hardy P. Practical and theoretical aspects of a method of orthodontic traction to unerupted teeth illustrated by three cases. Brit J Orthod, 13: 229-236, 1986.

[15]. Jena AK. Duggal R. Roychoudhury A. Parkash H. Orthodontic assisted tooth eruption in a dentigerous cyst: a case report. J Clin Pediatr Dent,29: 33-35, 2004.

[16]. Jones JW. Husain J. Management of the unerupted incisor. Dent Update, 23: 36-39, 1996. 